\title{
Diagnosis of interstitial lung disease by a percutaneous lung biopsy sample
}

\author{
Rosalind L Smyth, Helen Carty, Huw Thomas, Dick van Velzen, David Heaf
}

\begin{abstract}
A percutaneous lung biopsy sample was used to diagnose interstitial lung disease in nine children aged less than 42 months. Fibrosing alveolitis was diagnosed in eight children and Pneumocystis carinii pneumonia in one child. Complications associated with the procedure were minimal and the results of the biopsy sample enabled each child to be treated appropriately.

(Arch Dis Child 1994; 70: 143-144)
\end{abstract}

Interstitial lung diseases are rare but important causes of respiratory illness in infants and children, and one of the most common is fibrosing alveolitis, which has a reported death rate of $40 \%$ in one series of patients. ${ }^{1}$ In this disorder, clinical and radiographic features are not diagnostic and the severity of chest radiographic changes does not correlate with prognosis. ${ }^{1}$ In the diagnosis of interstitial lung disease in adults, Turner-Warwick has stressed the importance of taking a lung biopsy sample to enable early intervention with appropriate treatment and to guide prognosis. ${ }^{2}$ This is usually achieved by taking a transbronchial lung biopsy sample through a fibreoptic bronchoscope, a procedure which is possible in normal sized children older than 10 years. $^{34} \mathrm{~A}$ transbronchial biopsy sample has been taken via a rigid bronchoscope in children as young as 3 years, though there is a significant morbidity from large pneumothoraces. ${ }^{4}$

Fluoroscopically guided, a percutaneous needle biopsy is widely used in the diagnosis of focal lung lesions in adult patients with a high rate of correct diagnoses. ${ }^{5}$ We report our experience of the use of this technique, which we have adapted for the diagnosis of interstitial lung disease in infants and young children.

Dr Smyth.

Accepted 19 October 1993
Patients and methods

Between October 1988 and June 1992, nine children (mean age 10.5 months; range 1-42 months) had a percutaneous lung biopsy sample taken (table). All nine presented with respiratory distress and five were failing to thrive; on auscultation, crackles were present in seven and wheezes in four. Chest radiographs showed diffuse infiltrative shadows in all nine children. All but one child (patient 8) were well enough to tolerate computed tomography of the chest. These included $2 \mathrm{~mm}$ high resolution scans through the upper, middle, and lower zones. Appearances on the computed tomograms included cysts and bullae (5/8 children), consolidation ( $2 / 8$ children), and fibrosis ( $2 / 8$ children). Computed tomography was compatible with a diagnosis of interstitial lung disease in $7 / 8$ children, and appeared to exclude it in one child. In $2 / 7$ children the computed tomogram was compatible with a diagnosis of histiocytosis $\mathrm{X}$ and in three children with fibrosing alveolitis.

All biopsy samples were taken by one paediatric radiologist (HC) with the patient ventilated under general anaesthetic. The first three biopsy samples (patients 1-3) were taken using a Travenol aspiration cytology needle and the remainder using a 20 gauge disposable Biopty aspiration needle with a Biopty gun (Radi Medical Systems AB, Uppsala, Sweden). The biopsy site was selected from the area of maximum disease or infiltration, but avoiding major vessels or large cysts. Under fluoroscopic control the needle was advanced through the skin surface and the biopsy taken in arrested maximum inspiration. Two to three biopsy samples were taken through the same site with the needle angled in a slightly different projection. The samples were passed to the histopathology staff who confirmed, using an inverted microscope, that the biopsy sample received was lung tissue. Macroscopically, an adequate biopsy sample was a cone of tissue measuring about $1.5 \mathrm{~cm}$ long and $0.2 \mathrm{~cm}$ in diameter. No more than three biopsy samples were taken during any one procedure. After the procedure the chest was screened for evidence of haemorrhage or pneumothorax. The patient was observed closely on the ward for 24 hours after the biopsy sample had been taken and a chest radiograph was performed if the child developed increased respiratory distress.

Biopsy samples were fixed in $4 \%$ phosphate buffered $0 \cdot 1 \mathrm{M}$ formaldehyde $(\mathrm{pH} 7 \cdot 4)$ and were processed in a Shandon hypercentre overnight. Serial sections $(2 \mu \mathrm{m})$ were stained with haemotoxylin and eosin, Masson's trichrome, and Verhoeff's elastic van Giesson. 
Pneumocystis carinii was shown using Grocott's methenamine-silver nitrate stain and immunocytochemically using a monoclonal antibody and the avidin biotin alkaline phosphatase technique. Microscopically a biopsy specimen was considered adequate if it contained at least 30-40 sacculae and their associated alveoli and at least 10 smaller intersaccular septae. In all instances at least one adequate biopsy sample was obtained and there was no problem in fixing and staining the material.

\section{Results}

A histological diagnosis was made in all nine children (table). In eight this diagnosis was considered to be part of the spectrum of fibrosing alveolitis as described by Scadding, ${ }^{6}$ appearances varying from a predominantly cellular pattern of desquamative interstitial pneumonitis to one of progressive fibrosis. Corticosteroid treatment was started and resulted in a clinical response in all eight children. Seven of these eight children have been followed up for more than two years after the initial diagnosis. At their two year review, 4/7 children continued to require steroids by mouth, and one of these was receiving continuous oxygen treatment.

The ninth child (patient 8) had a biopsy sample taken one week after admission with what was thought to be opportunistic pneumonia. The child's clinical condition had deteriorated rapidly after admission and at the time the biopsy sample was taken he was dependent on a ventilator and required $100 \%$ oxygen. The lung biopsy sample showed a desquamative interstitial pneumonitis and immunohistological stains showed evidence of $P$ carinii infection. Treatment with intravenous high doses of co-trimoxazole was started and the child was subsequently found to have acquired immunodeficiency syndrome. The child died from the respiratory complications of $P$ carinii pneumonia two weeks after the lung biopsy sample was taken.

Complications (table) did not lead to a clinical deterioration in any child. One child, who had multiple lung cysts, developed a 20\% pneumothorax which resolved in two days without thoracocentesis. Shortly after this it was decided to use the smaller gauge Biopty aspiration needle with a Biopty gun. Small $(<10 \%)$ pneumothoraces arose in a further four patients, but resolved within 24 hours. Patient 8 , the only child to require ventilation at the time of the lung biopsy sample was taken, did not have any complications.

\section{Discussion}

This study has shown that taking a percutaneous needle biopsy sample of the lung may be useful in the diagnosis of interstitial lung disease in young children. As patient 8 shows, this procedure may also provide histological diagnosis of opportunistic pneumonia in the immunosuppressed infant.

The alternative to obtaining biopsy material for the histological diagnosis of interstitial lung disease is to base a diagnosis on clinical and radiographic features, with the knowledge that certain interstitial lung diseases are much more common than others. This approach has been condemned in adults ${ }^{2}$ and in our view is also unsatisfactory in the management of children. In the diagnosis of opportunistic pneumonia, there are technical difficulties in obtaining bronchoalveolar lavage in young children, but it is possible that non-bronchoscopic bronchoalveolar lavage may be developed for this age group in the future. ${ }^{7}$

At less than 3 years of age the only other means of obtaining lung tissue is by an open lung biopsy sample. This requires thoracotomy, a procedure in which the incidence of postoperative pneumothorax is so high that most surgeons recommend an intercostal chest drain after the operation. ${ }^{8}$ Taking a percutaneous lung biopsy sample is a simpler procedure which can be performed by a radiologist experienced in the use of such techniques, and the same operator is also able, under fluoroscopic control, to select the most suitable site for a biopsy sample. In this series the biopsy samples were adequate to make a diagnosis in every patient and most children were able to go home the day after the procedure had been carried out.

1 Hewitt CJ, Hull D, Keeling JW. Fibrosing alveolitis in infancy and childhood. Arch Dis Child 1977; 52: 22-37. Turner-Warwick MEH. Approaches to treatment of interstitial lung disease. Postgrad Med $\mathcal{F} 1984 ; 60$ : 183-6.

3 Scott JP, Higenbottam TW, Smyth RL, et al. Transbronchial biopsies in children after heart-lung transplantation. Pediatrics 1990; 86: 698-702.

4 Whitehead B, Scott JP, Helms P, et al. Technique and use of transbronchial biopsy in children and adolescents. Pediatr Pulmonol 1991; 12: 240-6.

5 Wescott JL. Direct percutaneous needle aspiration of localized pulmonary lesions: results in $\mathbf{4 2 2}$ patients. Radiology $1980 ; 137$ : 31-5.

6 Scadding JG. Lung biopsy in the diagnosis of diffuse lung disease. $B M 7$ 1970; ii: 557-64.

7 Alpert BE, O'Sullivan BP, Panitch HB. Non-bronchoscopic approach to bronchoalveolar lavage in children with artificial airways. Pediatr Pulmonol 1992; 13: 38-41.

8 Venn G. Open lung biopsy. Br f Hosp Med 1988; 39: 272-6. 